\title{
Brecha de género en la participación laboral y el tipo de unión conyugal en Argentina
}

\section{Gender gap in labor participation and type of marital union in Argentina}

Jorge A. Paz*

\section{Resumen}

En este artículo se analiza el posible efecto de la división de tareas dentro de los hogares conformados por parejas unidas y casadas sobre sus niveles e intensidades de participación en el mercado de trabajo. Con datos del Módulo de Uso del Tiempo, añadido a la Encuesta Anual de Hogares Urbanos del año 2013, se explora empíricamente la siguiente hipótesis: los hogares formados por parejas unidas distribuyen las tareas domésticas (no remuneradas) más igualitariamente que aquellos de parejas casadas, lo que conduce a una participación laboral más parecida tanto en incidencia como en intensidad de participación. Dicho de otra manera, mujeres y

* Universidad de Salta, Instituto de Estudios Laborales y del Desarrollo Económico. Dirección: Bolivia 5150 (A4408FVY), Salta, Argentina. Correo: pazjor@ gmail.com ORCID: https://orcid.org/0000-0002-2009-680X.

Nota del autor: Una versión previa de este artículo puede encontrarse en Paz (2018). Agradezco los comentarios recibidos de las personas que leyeron y comentaron aquel documento: Paulo Saad, Roberto Giuliodori, Leandro González y Enrique Peláez. También estoy en deuda con tres revisores anónimos que realizaron invalorables aportes a la versión inmediata precedente del trabajo que aquí se presenta. 
hombres casados tienden a una especialización mayor y, por lo tanto, se observa en estas parejas una brecha de participación entre géneros más amplia que la registrada entre mujeres y hombres unidos de hecho.

Palabras clave: usos del tiempo; género; participación económica; Argentina.

\section{Abstract}

This article analyzes the possible effect of task division within households made up of united and married couples, on their levels and intensities of participation in the labor market. With data from the Time Use Module, added to the Annual Survey of Urban Households (2013), the following hypothesis is empirically explored: households made up of unmarried couples actually distribute domestic tasks (unpaid) more equally than households of married couples do, which leads to a more similar labor participation both in incidence and in intensity of participation. In other words, men and women who are members of the latter tend to be more specialized and, therefore, a gap of participation between genders is observed in these couples that is wider than that registered between men and women in fact united.

Keywords: uses of time; gender; economic participation; Argentina.

\section{Introducción}

La participación de las mujeres en el mercado de trabajo ha recibido atención en numerosos estudios académicos y, más recientemente, entre quienes fungen como responsables del diseño e implementación de las políticas públicas. ${ }^{1}$ Particularmente, el interés ha girado en torno al nivel comparativamente bajo de actividad que registran las mujeres en comparación con los hombres.

${ }^{1}$ En Torns (2004) aparece ya un planteamiento de esta naturaleza, que gira en torno al tiempo y a la necesidad de su consideración en las políticas públicas. 
Por el lado académico, el interrogante principal tiene que ver con las causas que generan esa disparidad y la factibilidad de menguarla o anularla, si se considera que refleja desigualdades discriminatorias de acceso al mercado laboral. ${ }^{2}$ A partir de tal brecha, desde la política pública se ha planteado la posibilidad de crear un "dividendo de género" (DG) o "bono de género" (BG), del que dispondrían los países para generar crecimiento económico y contribuir, entre otras beneficios, a la disminución de la pobreza y la desigualdad económica. Lógicamente, el DG sería el resultado de políticas públicas apropiadas no sólo para fomentar la participación económica de las mujeres bajo consideraciones estrictas de equidad de género (Martínez, Miller y Saad, 2013), sino también para promover políticas de trabajo decente que incorporen estas consideraciones. La Organización Internacional del Trabajo ha calculado que cerrar la brecha de participación entre géneros provocaría un aumento del $5.3 \%$ del empleo mundial y que esto se produciría principalmente en los denominados "países emergentes" dado que es allí donde es más alta. Además, este resultado podría reportar importantes beneficios económicos y un incremento de 3.9 puntos porcentuales del PIB mundial para la proyección hacia el año 2025 (ILO, 2017).

Volviendo al interés académico, hay cierto acuerdo en la literatura de que una de las razones que explicaría la brecha de género en la participación económica es la especialización de los miembros de las parejas en la realización de determinadas actividades. Varias encuestas sobre usos del tiempo están mostrando que en América Latina el porcentaje de mujeres ocupadas en actividades no remuneradas es mayor que el de los hombres dedicado a ese tipo de tareas. Es decir, se sabe que las mujeres "inactivas" trabajan, pero al no hacerlo por un pago, son invisibles en las cuentas nacionales de sus respectivos países (Abraham y Mackie, 2005; Stiglitz, Sen y Fitoussi, 2009). El cuidado de niñas, niños y de personas mayores en el hogar aparece

${ }^{2}$ La literatura sobre este tema es voluminosa y particularmente creciente desde el momento en que la participación femenina en actividades remuneradas comenzó a aumentar en los países industrializados, cerca de los años ochenta. En realidad, la tendencia es creciente desde el primer dato disponible, correspondiente al año 1890 en Estados Unidos; crece a saltos más pronunciados desde 1960, y en 1980 supera el umbral del 50\% (Killingsworth y Heckman, 1986). 
en este contexto como tarea particular de la que se hacen cargo principalmente las mujeres, entre otras tantas labores del hogar que no son remuneradas. Si la brecha de género en la participación económica de la población está determinada, aunque no sea más que en parte, por la especialización en la realización de tareas dentro de los hogares, un reparto más igualitario de las responsabilidades hogareñas podría conducir a una reducción de tal brecha sin que medie, por ejemplo, una caída en la fecundidad.

El objetivo principal de este artículo es identificar el efecto de la especialización de las tareas en el interior de los hogares sobre la participación laboral de hombres y mujeres en Argentina. Se profundiza además en el análisis de los determinantes de otros posibles usos del tiempo, como el dedicado a las tareas domésticas no remuneradas (TDNR). Para identificar dicho efecto, se usa la variable estado civil, diferenciando las parejas de hogares nucleares casadas de las que se encuentran en unión consensual. El argumento que justifica esta manera de proceder tiene que ver con la estabilidad de la pareja, que implica uno u otro tipo de unión. En este sentido, se supone que el contrato matrimonial conlleva una estabilidad mayor que el simple acuerdo que rige en una pareja unida por consenso.

Hay una creciente literatura que se viene ocupando de este tema y cuyos resultados muestran que los miembros de las parejas unidas distribuyen más igualitariamente el tiempo del que disponen para realizar actividades. ${ }^{3}$ Con excepción de algunos aportes puntuales, ${ }^{4}$ se ha indagado menos sobre las razones que subyacen a esta mayor igualdad en la distribución de tareas, surgiendo de esta manera una serie de preguntas: ¿los hombres casados realizan menos tareas domésticas que sus pares unidos consensualmente? o ¿son las mujeres unidas las que destinan una mayor parte de su tiempo al mercado la-

${ }^{3}$ Pueden mencionarse los aportes de Blair y Lichter (1991), Shelton y John (1993) y South y Spitze (1994) como seminales de una literatura que creció notablemente desde entonces, principalmente impulsada por el proceso de "desinstitucionalización" del matrimonio ocurrido en las últimas décadas (Cherlin, 2004) en varios países, así como por la mayor y mejor calidad de los datos disponibles provenientes de las encuestas de uso del tiempo.

${ }^{4}$ Por ejemplo, Domínguez-Folgueras (2012a), quien destina buena parte de su contribución al examen de este tema. 
boral? Las respuestas que pueden darse a estas preguntas y otras similares tienen consecuencias no triviales. Es probable, por ejemplo, que la mayor participación económica de las mujeres unidas comparadas con sus pares casadas se deba a que asumen más tareas de ambos tipos, persistiendo, por ejemplo, las desigualdades en la distribución de las tareas dentro de los hogares. Mirar los acuerdos laborales que se dan en el seno de los hogares resulta fundamental para entender, aunque no sea más que en parte, las diferencias en el mercado de trabajo y en la asignación del tiempo en las tareas domésticas no remuneradas.

Este documento está organizado de la siguiente manera. En la próxima sección se desarrolla la argumentación que sostiene la indagación empírica de las secciones posteriores. En la sección III se repasan los estudios que han tratado este tema, poniendo énfasis en aquellos que se ocupan de las temáticas afines a la de la presente investigación. En la IV se describen los datos y se presenta la metodología seleccionada para su tratamiento. En la V se discuten los resultados del análisis, y en la VI se plantean algunas consideraciones generales que surgen de la discusión previa. El artículo contiene además un anexo de cuadros que sirven de soporte a algunas afirmaciones realizadas en su cuerpo central.

\section{La hipótesis explorada}

La hipótesis explorada en este artículo es la siguiente: una parte de la brecha de participación laboral entre hombres y mujeres está dada por la especialización de tareas dentro de los hogares, y dicha especialización difiere según el tipo de unión conyugal de las parejas. La importancia de este último factor comenzó a ser considerada en la investigación sobre estos temas no hace demasiado tiempo.

La teoría económica tradicional sostiene que la especialización en cualquier ámbito se produce si existen ventajas comparativas absolutas del intercambio entre los actores que se especializan, sean países, regiones de una nación, o personas dentro del hogar. Si se extiende esta idea a las ventajas que ofrece el matrimonio, puede decirse que, bajo determinadas circunstancias, la especialización de los miembros 
de una pareja puede mejorar el bienestar de ambos por varios motivos (por ejemplo, en una pareja heterosexual, la capacidad de las mujeres de mantener la gestación del feto, dar a luz y, en la mayoría de los casos, alimentar al recién nacido, es una diferencia biológica que les da cierta "ventaja" respecto a los hombres). Una prerrogativa importante es el aumento de la productividad individual de cada miembro, que aumenta a medida que la especialización se profundiza.

Afirmaciones como las precedentes conducen a Becker (1981) a incorporar el concepto de "ventaja comparativa" al estudio del uso del tiempo, planteando que la eficiencia en el funcionamiento de un hogar depende de que cada miembro se ocupe más de las actividades en las que es más productivo; si esto ocurre, la producción total del hogar resultará mayor que la que podría obtenerse sin especialización (Becker, 1965; Blau, Ferber y Winkler, 2014).

Así, los primeros aportes a la economía de la asignación del tiempo provienen de la teoría neoclásica de la oferta laboral: desde el modelo tradicional microeconómico en el que el individuo decide si trabajar o no, y en el caso de trabajar con qué intensidad hacerlo (Gronau, 1976), hasta los aportes más recientes que consideran al hogar como unidad de producción, más que de consumo (Browning, Chiappori y Weiss, 2014; Bryant y Zick, 2006). En esta dirección, se supone que los hogares utilizan el tiempo y los bienes como factores para producir algunas mercancías que proporcionan utilidad a sus miembros.

Los impactos de la fecundidad, la presencia de personas mayores y el tiempo dedicado a las tareas de cuidado sobre la participación económica femenina pueden ser ubicados en este contexto teórico (Killingsworth y Heckman, 1986). Si es la mujer la que se ocupa de estas tareas, un aumento en el número de niños o de las personas a su cargo en general, supone un incremento en la demanda del tiempo dedicado al cuidado $y$, en consecuencia, menor tiempo disponible para el trabajo remunerado. Esto puede desembocar en la restricción de sus derechos, limitar sus capacidades y obstaculizar el avance hacia la igualdad de género (Esplen, 2009). De esta forma, el salario que la mujer debería percibir para compensar el costo en tiempo de las tareas de cuidado de sus hijos sería mayor cuanto más grande sea el número de hijos que tenga. 
Es necesario aquí diferenciar las actividades de cuidado del resto de las tareas domésticas (Aguirre, García Sainz y Carrasco, 2005; García, 2019). El concepto de cuidado puede incluir no sólo la asistencia que reciben las personas con necesidades específicas (niños, ancianos y enfermos), sino también la que se proporciona a adultos sanos al preparar la comida, lavar su ropa y hasta hacer sus compras. Estas actividades pueden ser remuneradas cuando la realizan trabajadores domésticos, enfermeros y "caseros" (trabajadores de casas de familia que desempeñan labores de jardinería, vigilancia, reparaciones, etc.); o no remuneradas, cuando el responsable es un familiar o un voluntario de la comunidad. Es en este último caso en el que se habla de TDNR.

Desde una perspectiva empírica, el impacto de las actividades de cuidado, en su sentido amplio, no está siendo captado por las variables tradicionales que suelen usarse para estudiar la participación femenina, como el número de hijos y de personas mayores en el hogar. Resulta necesario, entonces, buscar alternativas que permitan una aproximación más precisa al problema. Claramente, son varios los factores que inciden en las personas en el momento de decidir cómo asignar su tiempo entre diferentes actividades: el estado civil, la educación, la cantidad de hijos, la condición laboral, la cultura, el nivel de ingresos, entre otros. Para Bloemen, Pasqua y Stancanelli (2010), hasta el grado educativo de los padres juega un papel importante en esta decisión. No todas estas variables están disponibles en las bases de datos que se disponen para el análisis empírico del problema.

Pero, a diferencia de las encuestas tradicionales, las de uso del tiempo han permitido avanzar firmemente en esta dirección. Un documento publicado por el Instituto Nacional de las Mujeres de México, con datos la Encuesta Nacional de Uso del Tiempo de 2009, afirma que las mujeres asignan 27 horas semanales más que los hombres al trabajo no remunerado, y que los hombres registran, en promedio, 19 horas semanales más que ellas al trabajo en el mercado. Con todo, las mujeres alcanzan 59 horas de trabajo total (remunerado y no remunerado) por semana, mientras que los hombres, 51. Bloemen, Pasqua y Stancanelli (2010), por su parte, presentan datos de 15 países de Europa para el periodo 2002-2003; en 13 de ellos las mujeres registran más horas de trabajo total que los hombres. Las excepciones son 
Suecia, donde los varones superan a las mujeres por cuatro horas, $\mathrm{y}$ Noruega, en donde ellas y ellos trabajan la misma cantidad de horas.

Las mujeres de los países analizados por Bloemen, Pasqua y Stancanelli (2010) tienen baja fecundidad, lo que refuerza la idea de que la presencia de infantes en el hogar es un determinante clave de la participación de las mujeres en el mercado de trabajo. Por ejemplo, se ha afirmado que la expansión de la tasa de actividad femenina que tuvo lugar en América Latina entre 1980 y 2010 tuvo que ver con la caída de la fecundidad (Martínez, Miller y Saad, 2013) y la consecuente asunción de mayor carga del tiempo total de trabajo entre las mujeres. Sin dejar de ser cierto, se plantea aquí que el efecto de la fecundidad sobre la participación económica está condicionado al tiempo que cada miembro de una pareja destine al cuidado de los niños y a la existencia de políticas públicas de cuidado con consideraciones de equidad de género. Una distribución más igualitaria del tiempo de cuidado entre los sexos, o una mayor disponibilidad de servicios profesionales de cuidado, pueden provocar una expansión de la actividad económica femenina sin que cambie la fecundidad, como ocurre, por ejemplo, con la comparativamente alta fecundidad de las mujeres suecas (Hoem, 2005).

\section{Literatura pertinente}

En esta revisión se pondrá énfasis en textos que evalúan la participación laboral de hombres y mujeres con pareja, específicamente en las diferencias entre quienes están casados y quienes cohabitan (unión consensual), pues es un importante indicador de especialización de tareas dentro de los hogares. En este sentido, las parejas casadas (en matrimonio) tienen un incentivo mayor a especializarse que las unidas porque, entre otras cosas, ante una presunta disolución, la existencia de un contrato asegura ciertas pautas de reparto de bienes, las cuales estarían ausentes para las parejas unidas consensualmente. Por este motivo, en este último tipo de parejas no habría incentivos a la especialización (al menos en el sentido aludido), tendiendo sus miembros a diversificar el uso de su tiempo y a especializarse menos que las parejas casadas. 
García y De Oliveira (2007) advierten sobre la importancia que tienen las desigualdades de la división sexual del trabajo en las parejas y ubican esas disparidades al nivel de las laborales, como la segregación y la discriminación. Esto conduce a mirar lo que ocurre dentro del hogar y a seleccionar indicadores que permitan dar cuenta de la división sexual del trabajo y de la especialización en los hogares.

En esa dirección, el estudio de Henkens, Grift y Siegers (2002) muestra que, en Holanda, las características de las mujeres casadas y las unidas son similares, pero ellas se comportan de manera diferente ante los mismos condicionantes. Davis, Greenstein y Gerteisen (2007) incorporan a los hombres al análisis de la oferta de trabajo y encuentran que los unidos realizan más trabajo doméstico que los casados, y que las mujeres unidas realizan menos trabajo doméstico que las casadas. Los autores hallan también que la disponibilidad de tiempo y los recursos de ambos tipos de uniones son sustancialmente los mismos (al igual que se anota en el estudio de Henkens, Grift y Siegers, 2002). Sostienen también que la especialización es mayor en los hogares en los que el hombre es el principal proveedor de ingresos. Un efecto similar se observa cuando el varón trabaja un número mayor de horas fuera del hogar.

Hallazgos similares son analizados por Bianchi, Lesnard, Nazio y Raley (2014), para quienes las personas unidas por consenso están menos protegidas si la relación se disuelve. Claramente, si esto se cumple, la brecha de género en el trabajo remunerado y no remunerado entre las personas casadas y las que cohabitan debe ser mayor en los países donde la cohabitación está menos institucionalizada y donde los que cohabitan tienen relativamente menos protecciones legales si la relación se disuelve.

Un enfoque un tanto diferente a los anteriores es el que siguen Barg y Beblo (2012), quienes encuentran que las parejas que anticipan la especialización en el uso del tiempo estando en cohabitación, terminan en matrimonio legal. Otro impulso adicional a la especialización ocurre cuando las parejas se convierten en padres. Este tema es planteado por Baxter (2005), y lo retoman Barg y Beblo (2012), con algunos matices.

Esas diferencias en la asignación de tiempo entre los miembros de las parejas casadas y las que cohabitan están afectadas también 
por el tipo de tareas realizadas y por lo que cada país entiende por cohabitación. Domínguez-Folgueras (2012b) estudia particularmente esos determinantes en cinco países europeos y considera que ambos son factores cruciales al evaluar las diferencias entre cónyuges bajo distintos regímenes familiares. Ella encuentra que los hombres y las mujeres que cohabitan gastan menos tiempo en tareas domésticas. Es decir, la mayor igualdad no es resultado de que ellos realicen más tareas domésticas y las mujeres menos, sino es consecuencia de que ambos miembros de la pareja dedican menos tiempo a estas tareas.

Hay otros autores, como Arosio (2017) y Meggiolaro (2014), que arriban a conclusiones análogas a la de los otros estudiosos del tema, pero esta última autora agrega un elemento crucial: la experiencia de uniones anteriores en la distribución del tiempo dentro del matrimonio, ya que los matrimonios que han experimentado uniones previas distribuyen más igualitariamente el tiempo de trabajo doméstico. Sánchez Peña y Pérez Amador (2016) estudian el caso mexicano y acotan su análisis a las parejas con doble proveedor. Al igual que lo que arrojan las investigaciones sobre países de Europa, las autoras encuentran una menor especialización en los hogares cuyos cónyuges están en unión libre. Dado que la brecha de tiempo dedicado a TDNR se reduce conforme aumenta el ingreso, las parejas en unión libre y de alto ingreso representan el arreglo más igualitario en la división sexual del trabajo no remunerado en el interior de los hogares. El determinante próximo de este resultado es que las mujeres en unión libre dedican menos tiempo al trabajo doméstico que las casadas. Las autoras no encuentran diferencias entre los varones.

El Cuadro 1 resume de manera breve la literatura que se ha comentado en los párrafos anteriores. Como puede verse en el cuadro, los estudios realizados sobre la temática usan como variable a explicar la participación de hombres y mujeres en actividades remuneradas y no remuneradas, y el tiempo que dedican a unas y otras. Las variables explicativas centrales pasan por el tipo de unión, esto es, matrimonio o unión consensual; y las variables de control por la edad, el nivel educativo, las características del cónyuge, y la región o provincia de residencia. Según la variable a explicar, se aplican modelos de regresión ya sean lineales o no lineales, y en algunos casos se corrige por selección muestral. Los resultados son consistentes con la hipótesis de 
que existe una distribución más igualitaria del tiempo de trabajo remunerado y no remunerado entre las parejas unidas, comparadas con las casadas, lo que muestra que en cierta forma se verifican algunas de las hipótesis centrales del modelo de la especialización desarrollado en el marco conceptual.

\section{Cuadro 1}

Parte de la literatura que analiza la relación entre tipo de unión y usos del tiempo en hombres y mujeres

\begin{tabular}{|c|c|c|c|c|c|}
\hline Autor & País & $\begin{array}{l}\text { Unidad } \\
\text { análisis }\end{array}$ & $\begin{array}{c}\text { Variables } \\
\text { principales }\end{array}$ & $A \tilde{n} \boldsymbol{o}$ & Metodología \\
\hline $\begin{array}{l}\text { Henkens, Grift } \\
\text { y Siegers, } \\
2002\end{array}$ & Holanda & $\begin{array}{l}\text { Menores } 65 \\
\text { años }\end{array}$ & $\begin{array}{l}\text { Participación } \\
\text { y horas }\end{array}$ & 1989-1998 & Logit y $\mathrm{MCO}$ \\
\hline $\begin{array}{l}\text { Davis, } \\
\text { Greenstein } \\
\text { y Gerteisen, } \\
2007\end{array}$ & 28 países & $\begin{array}{l}\text { Hombres } \\
\text { y mujeres }\end{array}$ & $\begin{array}{l}\text { Participación } \\
\text { trabajo } \\
\text { doméstico }\end{array}$ & 2002 & $\begin{array}{l}\text { Modelos } \\
\text { multinivel }\end{array}$ \\
\hline $\begin{array}{l}\text { Kuperberg, } \\
2012\end{array}$ & EU & Parejas & $\begin{array}{l}\text { Participación } \\
\text { y horas }\end{array}$ & 2002 & Logit y $\mathrm{MCO}$ \\
\hline $\begin{array}{l}\text { Barg y Beblo, } \\
2012\end{array}$ & Alemania & Parejas & $\begin{array}{l}\text { Asignación } \\
\text { del tiempo }\end{array}$ & $1991-2008$ & Probit y PSM \\
\hline $\begin{array}{l}\text { Domínguez- } \\
\text { Folgueras, } \\
2012 \mathrm{a}\end{array}$ & España & Parejas & $\begin{array}{l}\text { Asignación } \\
\text { del tiempo }\end{array}$ & $2002-2003$ & $\mathrm{MCO}$ \\
\hline $\begin{array}{l}\text { Domínguez- } \\
\text { Folgueras, } \\
2012 \text { b }\end{array}$ & $\begin{array}{l}5 \text { países } \\
\text { Europa }\end{array}$ & Parejas & $\begin{array}{l}\text { Asignación } \\
\text { del tiempo }\end{array}$ & $2002-2003$ & $\mathrm{MCO}$ \\
\hline $\begin{array}{l}\text { Bianchi et al., } \\
2014\end{array}$ & $\begin{array}{l}\text { Francia, } \\
\text { Italia y EU }\end{array}$ & $\begin{array}{l}\text { Hombres } \\
\text { y mujeres }\end{array}$ & $\begin{array}{l}\text { Participación } \\
\text { en TR y TNR }\end{array}$ & 2003-2009 & Logit y $\mathrm{MCO}$ \\
\hline $\begin{array}{l}\text { Meggiolaro, } \\
2014\end{array}$ & Italia & Parejas & $\begin{array}{l}\text { Asignación } \\
\text { del tiempo }\end{array}$ & 2003 & $\mathrm{MCO}$ \\
\hline $\begin{array}{l}\text { Sánchez } \\
\text { Peña y Pérez } \\
\text { Amador, } 2016\end{array}$ & México & Parejas & $\begin{array}{l}\text { Asignación } \\
\text { del tiempo }\end{array}$ & 2010 & MCO y CSS \\
\hline Arosio, 2017 & Italia & $\begin{array}{l}\text { Hombres y } \\
\text { mujeres }\end{array}$ & $\begin{array}{l}\text { Asignación } \\
\text { del tiempo }\end{array}$ & 2012 & $\mathrm{MCO}$ \\
\hline
\end{tabular}

Nota: $\mathrm{CSS}=$ corrección por sesgo de selección; $\mathrm{TR}=$ trabajo remunerado; $\mathrm{TDNR}=$ trabajo doméstico no remunerado; $\mathrm{MCO}=$ mínimos cuadrados ordinarios; $\mathrm{PSM}=$ propensity score matching.

Fuente: Elaboración propia con base en las fuentes anotadas. 


\section{Datos y estrategia metodológica}

\section{A. Datos}

En esta investigación se usaron datos del Módulo sobre Trabajo No Remunerado y Uso del Tiempo (MUT), levantado junto con la Encuesta Anual de Hogares Urbanos (EAHU) durante el tercer trimestre del año 2013 por el Instituto de Estadística y Censos (INDEC), con la colaboración de las oficinas provinciales de Estadística de Argentina. En el operativo EAHU 2013 se entrevistaron 65688 personas, que representan a una población de 26697022 individuos de 18 años y más de edad, residentes en hogares particulares de localidades de 2000 o más habitantes de todo el territorio nacional. El MUT recopiló información de 65352 personas de 18 años y más. Fueron excluidos de los hogares los residentes de servicio doméstico con cama.

El objetivo general del MUT fue obtener información sobre la participación y el tiempo destinado por las personas de 18 años y más a las tareas domésticas, al cuidado de los miembros del hogar y al trabajo voluntario. Sus objetivos más específicos fueron cuantificar: a) la magnitud del trabajo no remunerado (TNR); b) las desigualdades de género en el tiempo dedicado al trabajo no remunerado doméstico y de cuidados en el interior de los hogares; y c) las actividades que la población realiza gratuitamente, tanto para la comunidad como para otros hogares. Un objetivo adicional, pero no por ello menos importante, fue generar información para la cuenta satélite de los hogares que visualice el trabajo no remunerado.

El MUT tiene la gran virtud de ser el primer operativo de cobertura nacional en Argentina sobre usos del tiempo de la población, ${ }^{5}$ pero tiene también muchas limitaciones. Por ejemplo, no incluye preguntas acerca del tiempo destinado a la educación, al cuidado per-

${ }^{5}$ La Ciudad Autónoma de Buenos Aires cuenta con dos encuestas de uso del tiempo, con datos para los años 2005 y 2016. También hay una encuesta realizada por la Universidad Nacional de Rosario con el Instituto Provincial de Estadística y Censos que contiene datos de la ciudad de Rosario (provincia de Santa Fe), correspondientes al año 2010. No hay datos disponibles al público de esta última encuesta. 
sonal y al ocio, entre otras actividades, ni permite diferenciar el tipo específico de quehacer doméstico. Hay un gran grupo que incluye actividades diversas (tales como la limpieza de la casa, el aseo, el arreglo de ropa, la preparación de alimentos, las compras para el hogar, la reparación y el mantenimiento del hogar) que, se sabe, tienen cierta especificidad de género (Esquivel, 2009). El MUT tampoco consideró las actividades simultáneas.

\section{B. Estrategia metodológica}

La metodología seguida en este artículo se ordena teniendo en cuenta la pregunta central que se propone responder: ¿los hombres y mujeres en unión distribuyen más igualitariamente el tiempo dedicado a trabajar (tanto para el mercado como para el hogar), de manera tal que registran una brecha de participación laboral menor que la de los hombres y las mujeres en matrimonio?

Para responder ello, se avanza por etapas. En primer lugar, se revisa la información descriptiva (no condicional) para evaluar la brecha de género en la participación laboral tanto en incidencia (participa / no participa), como en intensidad (número de horas dedicadas al trabajo remunerado y a TDNR). En segundo lugar, se estiman modelos multivariados que tienen como objetivo responder interrogantes intermedios: a) ¿la brecha de participación laboral entre los miembros de las parejas unidas o casadas se debe a que los hombres dedican más / menos tiempo a las actividades no remuneradas (quehaceres domésticos, cuidado de personas, apoyo escolar)? o ¿son las mujeres las que dedican más / menos tiempo a las actividades de mercado que al trabajo doméstico no remunerado?; b) ¿en qué tipo de tareas no remuneradas se observan los diferenciales mayores de participación (incidencia y horas) en el trabajo doméstico no remunerado entre hombres unidos y casados y mujeres unidas y casadas?

Los modelos multivariados que permiten identificar el efecto de los arreglos matrimoniales en parejas heterosexuales sobre la incidencia (nivel) y la intensidad (horas) de la participación laboral de hombres y mujeres responden a la siguiente especificación: 


$$
P_{i} \text { ó } H_{i}=\beta_{0}+\beta_{1} A M_{i}+\sum_{j=4}^{m} \beta_{j} V C_{i}+u
$$

donde $P_{i}$ representa la probabilidad de un hombre o de una "i" de participar en el mercado laboral y $H_{i}$ el número de horas dedicadas al trabajo por un pago; $A M_{i}$ es el tipo de unión (unido / casado), y $V C_{i}$ las variables de control incluidas en el modelo. Los parámetros $\beta_{0}, \beta_{1}, \beta_{2}, \beta_{3}$ y $\beta_{j}$ deben ser estimados. Por su parte, $u$ representa el término de error.

Dado que se define $A M=1$ si la persona está casada, la hipótesis nula puede formularse así: $\beta_{1}=0$, lo que estaría significando que casados y unidos (hombres y mujeres) participan de manera igualitaria en el mercado laboral o que destinan el mismo número de horas al trabajo remunerado, controlados (condicional a) todos los demás factores incluidos en la regresión.

La diferencia de tratamiento dada a la probabilidad de participar $\left(P_{i}\right)$ y a las horas trabajadas $\left(H_{i}\right)$ se manifiesta en el tipo de modelo estimado. Dado que la primera es una variable dicotómica (toma valores 0 y 1), se estima un modelo probit. ${ }^{6}$ La segunda es una variable continua y se aplica un modelo de regresión lineal por mínimos cuadrados ordinarios (MCO). ${ }^{7}$ En ambos tipos de modelos todo se trata de la misma manera: se considera una independiente central (tipo de unión, $\left.A M_{i}\right)$ y variables de control $\left(V C_{i}\right)$.

Para el análisis del tiempo dedicado al segundo tipo de actividades, a TDNR, se estima un segundo grupo de regresiones y la especificación es similar al caso anterior (trabajo para el mercado). Además de la cantidad de horas semanales dedicadas al trabajo doméstico no remunerado total, se diferencia en este caso entre: a) quehaceres domésticos; $b$ ) apoyo escolar; y $c$ ) cuidado de personas.

${ }^{6}$ La alternativa más usada en los estudios comentados en la sección 3, Cuadro 1 , es el modelo logit. Se trata de alternativas lícitas.

${ }^{7}$ Una posibilidad es estimar un modelo tobit, dada la gran cantidad de casos con valor cero, principalmente entre las mujeres. Si bien esto permite aprovechar todos los datos de la muestra y corregir el sesgo que provoca en la estimación la presencia de ceros, Stewart (2009) ha mostrado de manera contundente la posible existencia de un sesgo en este tipo de modelos, por lo que aquí se sigue la estimación por MCO. 
La variable dependiente central y las de control son idénticas a las usadas para la estimación de los modelos con variables dependientes dicotómicas.

Las variables de control $\left(V C_{i}\right)$ son: la edad, la educación, la cantidad de niñas y niños en el hogar, la cantidad de personas mayores, y el monto de ingreso no propio. La elección de estas variables tiene que ver con lo discutido en el marco conceptual sobre la participación económica: algunas afectan el salario potencial (la educación, por ejemplo), otras operan más directamente sobre el salario de reserva (hijos y personas mayores, entre otras), incidiendo sobre las decisiones de participación y horas.

\section{Resultados}

En las zonas urbanas de Argentina, la brecha de participación laboral entre hombres y mujeres en edades centrales (18-64) es de 38 puntos porcentuales: $94 \%$ en hombres y $56 \%$ en mujeres (Cuadro 2 ). Si se considera el tipo de unión conyugal, se aprecia que tanto las mujeres como los hombres en unión consensual participan más que las y los casados, lo que hace similares las brechas entre hombres y mujeres unidas/os (96\% versus 58\%) y casadas/os (93\% versus 55\%).

\section{Cuadro 2}

Tasas de actividad por género (\%) y horas semanales trabajadas para el mercado por la población adulta (18-64), según situación conyugal. Argentina, 2013

\begin{tabular}{lccccccc}
\hline & \multicolumn{3}{c}{ Participación } & & \multicolumn{3}{c}{ Horas } \\
\cline { 2 - 3 } \cline { 6 - 8 } Género & Unidas/os & Casadas/os & Total & & Unidas/os & Casadas/os & Total \\
\hline Varón & 96.4 & 92.7 & 94.4 & & 46.4 & 45.6 & 46.0 \\
Mujer & 57.5 & 55.3 & 56.3 & & 30.9 & 32.7 & 31.9 \\
Total & 76.9 & 73.4 & 75.0 & & 40.8 & 40.7 & 40.8 \\
\hline
\end{tabular}

Fuente: Elaboración propia con datos de INDEC, EAUH.

En el Cuadro 2 se reporta también el promedio de horas trabajadas entre hombres y mujeres bajo distinto tipo de unión conyugal. La 
brecha general arroja 14 horas semanales de diferencia, mayor entre los hombres. Se observa que unidos y casados dedican el mismo número de horas al trabajo remunerado, mientras que las mujeres unidas trabajan dos horas más en promedio en el mercado. La diferencia en horas trabajadas por un pago es levemente inferior entre hombres y mujeres casadas/os, resultado opuesto a lo que se esperaba según la literatura revisada en la sección III.

El Cuadro 3 es idéntico al anterior, pero se reporta aquí la participación en el trabajo doméstico no remunerado (TDNR) y las horas semanales dedicadas a ese tipo de tareas.

\section{Cuadro 3}

Tasas de actividad (TDNR) por género (\%) y horas semanales trabajadas en el hogar por la población adulta (18-64), según situación conyugal. Argentina, 2013

\begin{tabular}{lccccccc}
\hline & \multicolumn{3}{c}{ Participación } & & \multicolumn{3}{c}{ Horas } \\
\cline { 2 - 3 } \cline { 7 - 8 } Género & Unidas/os & Casadas/os & Total & & Unidas/os & Casadas/os & Total \\
\hline Varón & 63.0 & 59.0 & 60.8 & & 17.4 & 16.0 & 16.6 \\
Mujer & 95.4 & 94.0 & 94.6 & & 56.1 & 47.8 & 51.5 \\
Total & 79.2 & 77.1 & 78.1 & & 36.8 & 32.5 & 34.4 \\
\hline
\end{tabular}

Fuente: Elaboración propia con datos de INDEC, EAUH.

En este caso, se aprecia que las diferencias entre géneros son similares en tamaño a las presentadas en el Cuadro 2, pero se dan en sentido opuesto: la participación de las mujeres supera en 34 puntos porcentuales a la de los hombres, y aquéllas trabajan 35 horas semanales más que éstos en tareas domésticas. Tal es el patrón encontrado en todas las investigaciones que se ocuparon del tema y que se remontan a mediados de los años noventa, cuando algunos países comenzaron a indagar este tipo de cuestiones. ${ }^{8}$

La disparidad de participación es mayor entre casadas y casados que entre unidas y unidos, pero la brecha en el número de horas semanales es mayor para unidas y unidos. Esto se debe a que el prome-

${ }^{8}$ Por ejemplo, Baxter (2005) muestra que éste es el caso de mujeres y hombres australianas/os en una encuesta nacional en Australia entre los años 1996 y 1997. 
dio de horas dedicadas a las tareas domésticas de las mujeres unidas supera en casi diez horas al de las mujeres casadas. En principio, éste es un hecho contraintuitivo y se aleja claramente de la hipótesis de una distribución más igualitaria del tiempo entre hombres y mujeres unidas/os y de la literatura pionera sobre el tema (Baxter, 2005). No obstante, dicha diferencia puede estar afectada por otras variables que no están siendo controladas en este examen descriptivo.

Justamente, para develar el efecto neto del tipo de unión conyugal sobre la participación económica y las horas dedicadas al mercado de trabajo, es necesario estimar los modelos multivariados descritos en el aparato B de la sección IV de este artículo. Todos los resultados de esas estimaciones se reúnen en el Anexo. A continuación, se resumirán los principales hallazgos, siguiendo el siguiente orden: en el apartado A se comentan los resultados de la incidencia de la participación tanto en actividades realizadas para el mercado como en tareas domésticas no remuneradas. El apartado B se ocupa de las diferencias entre casadas/os y unidas/os en la intensidad de la participación, medida en horas semanales. Por último, el apartado $\mathrm{C}$ examina las diferencias encontradas entre hombres y mujeres casadas/os y unidas/os en dos actividades domésticas específicas: los quehaceres domésticos y las tareas de cuidado de personas. Para el examen de este último tema, la muestra se restringe a los hogares en los que hay niñas, niños y personas mayores.

En el Cuadro A1 del Anexo se presentan los estadísticos descriptivos que permiten formarse una primera idea de la situación de Argentina en el tema tratado. Así, el 57\% de la población urbana argentina de 18 años y más está casada, siendo mayor el porcentaje entre las mujeres; ellas tienen un nivel educativo superior al de los hombres; hay más niños que personas mayores en los hogares; y entre los infantes, el grupo más numeroso es el comprendido entre los 5 y los 12 años. Una variable particularmente descriptiva de la división del trabajo en el interior de los hogares es el ingreso no propio: nótese que el de las mujeres es 1.7:1 frente al de los hombres, en lo que claramente se encuentra el efecto de la falta de valuación de TDNR. 


\section{A. Incidencia de la participación}

Los determinantes de la participación en el mercado laboral se muestran en el Cuadro A2 del Anexo, mientras que los correspondientes a TDNR aparecen en el Cuadro A3. En el primero puede apreciarse que hombres y mujeres casados acusan una participación laboral menor que hombres y mujeres unidos. Pero se constata fácilmente que la disparidad proviene de la participación de las casadas y las unidas, dado que, para los hombres, no puede rechazarse la hipótesis nula de igual nivel entre casados y unidos. En suma, ante un nivel de participación menor de casadas y un nivel similar entre hombres bajo los dos tipos de unión conyugal, la disparidad de participación entre casadas y casados es mayor que la registrada entre unidas y unidos. Este resultado va en línea con lo encontrado en otros estudios (Arosio, 2017; Bianchi, Lesnard, Nazio y Raley, 2014).

El resto de las variables de control se comportan de acuerdo a lo esperado; esto es, un perfil por edad en forma de "U" invertida: la participación laboral de hombres y mujeres es baja en edades extremas (jóvenes y personas mayores) y alta en las centrales. La educación impacta positivamente en la probabilidad de participación, pero lo hace más en las mujeres que en los hombres. La presencia de niñas, niños y personas mayores en el hogar reduce la participación de las mujeres, pero, en general, no modifica la de los hombres. Un caso curioso se da entre niñas y niños menores de cinco años: es el grupo que provoca el impacto más fuerte sobre la participación laboral de las mujeres y afecta también la de los hombres, pero de manera opuesta: aumentándola.

El Cuadro A3 del Anexo, por su parte, sugiere que no hay razones sólidas para sostener que las personas con distintos tipos de unión conyugal (tanto hombres como mujeres) participen más o menos en actividades o tareas domésticas no remuneradas (TDNR). Dicho de otra manera, la participación en este tipo de actividad entre personas de diferente tipo de unión conyugal es similar en TDNR.

Las otras variables incluidas en los modelos operan también con el signo esperado. La edad, en este caso, no ejerce efecto sobre la participación en TDNR. Los hombres más educados participan más en dichas tareas, mientras que las mujeres más educadas, menos. De 
las personas más estrictamente ligadas al cuidado de niñas, niños y personas mayores, se aprecia que son las mujeres las que acusan el efecto mayor, aunque también surge significatividad en el caso de los varones. Las personas mayores no ejercen efecto significativo ni en hombres ni en mujeres.

\section{B. Determinantes de la intensidad de la participación laboral y en TDNR}

Las diferencias de intensidad en horas semanales dedicadas al trabajo remunerado encontradas (Cuadro A4 del Anexo) permiten afirmar que la falta de significancia entre personas de ambos sexos unidas/os y casadas/os se debe a la dirección en la que opera la relación para cada género por separado. Entre los hombres, los casados trabajan un número mayor de horas para el mercado que los unidos; mientras que, entre las mujeres, las casadas trabajan un número menor de horas para el mercado que las unidas. El signo opuesto hace que la brecha general en las parejas casadas sea mayor que en las unidas. Este resultado va en línea con lo encontrado en estudios similares: hombres y mujeres unidos distribuyen más igualitariamente la participación en el mercado de trabajo. Si este fenómeno se asocia a la especialización, se estaría observando mayor incremento de ésta en las parejas casadas que en aquellas que están en unión consensual.

No obstante, hay que advertir que puede existir un sesgo importante que no se puede corregir con los datos disponibles en este trabajo y que puede afectar las comparaciones. Como lo muestra $\mathrm{Ku}-$ perberg (2012), las comparaciones generales no capturan adecuadamente el rango de comportamiento que se verifica en la convivencia (parejas unidas) y el matrimonio. Si bien esta autora encuentra evidencias claras de mayor especialización dentro del matrimonio (similar a lo encontrado aquí para Argentina), destaca que la conducta de los miembros de las parejas unidas, pero que tienen planes de matrimonio, no se diferencia de aquella típica de miembros de parejas casadas, pero recientemente. El hallazgo de esta autora es importante, dado que implica que el efecto causal del matrimonio en el com- 
portamiento de los miembros de la pareja puede acumularse con el tiempo de casados.

No obstante, cuando se examina el papel de las horas semanales dedicadas a TDNR, la conclusión cambia: las mujeres casadas destinan casi dos horas más por semana al desarrollo de TDNR que las unidas, mientras que entre hombres casados y unidos no se encuentran disparidades significativas (véase el Cuadro A5 en el Anexo). Estos resultados coinciden sólo en parte con la literatura internacional, ya que en otros países se encuentran diferencias significativas entre unidas/os y casadas/os (Bianchi, Lesnard, Nazio y Raley, 2014; Davis, Greenstein y Gerteisen, 2007; Henkens, Grift y Siegers, 2002).

Este resultado cambia las conclusiones del análisis descriptivo realizado anteriormente (Cuadro 2), pues las mujeres unidas aparecían allí dedicando más tiempo a TDNR que las casadas. Claramente, esos promedios están afectados por las variables que se controlan en el análisis condicional, cuyos resultados se muestran en el Cuadro A5. Algo similar ocurre en el estudio realizado por Henkens, Grift y Siegers (2002), que se corrige cuando se controla por el monto de ingreso que aportan hombres y mujeres al hogar.

Si se conectan los resultados de los Cuadros A3 y A5 del Anexo, puede arriesgarse la siguiente afirmación: las mujeres casadas destinan menos tiempo al mercado laboral porque lo vuelcan a TDNR. Pero nótese que el intercambio en tiempo no es simétrico: las mujeres casadas destinan 0.8 horas menos por semana al mercado laboral y 1.9 horas más al trabajo doméstico. Esto evidencia una cantidad mayor de horas netas dedicadas a ambas actividades en conjunto y una sobrecarga de trabajo para las mujeres casadas. Este hallazgo concuerda con la literatura que sostiene la doble carga laboral que enfrentan las mujeres tanto dentro como fuera de casa y que la interpreta como evidencia a favor de la hipótesis de la especialización (Domínguez-Folgueras, 2012a).

Del resto de las variables incluidas en las regresiones, puede verse lo siguiente: las horas trabajadas para el mercado (Cuadro A4 del Anexo) no resultan sensibles a la mayoría de las variables independientes consideradas, excepto a la presencia de niñas y niños menores de trece años. Esto quiere decir que el número de niñas y niños no 
sólo reduce la participación, sino que también saca horas semanales de trabajo a aquellas que participan. No es el caso de las personas mayores.

Por el contrario, las horas dedicadas a TDNR (Cuadro A5 del Anexo) resultan altamente sensibles al resto de las variables independientes incluidas en los modelos. En este caso, pueden apreciarse las brechas de género en las tareas específicas de cuidado de personas. También surge con claridad que son las mujeres las que se hacen cargo de las personas mayores presentes en el hogar, aun en los casos en los que se aprecia un comportamiento masculino involucrado con el cuidado: por ejemplo, se observa que los hombres que residen en hogares en los que hay mayores de 80 años dedican 2.4 horas a TDNR, mientras que las mujeres dedican 8.9 horas semanales más. Esto indica una diferencia casi cuatro veces mayor.

\section{Incidencia e intensidad sobre tareas domésticas no remuneradas}

Es relevante diferenciar los diferentes tipos de tareas realizadas por hombres y mujeres dentro del hogar, tanto en lo que atañe a los quehaceres domésticos como a los cuidados (García, 2019) y a las tareas de apoyo escolar. Son sólamente éstas las TDNR que pueden apreciarse con claridad con los datos disponibles en el MUT de la EAUH. Esa diferencia aparece claramente en el Cuadro A6a (mujeres) y en el Cuadro A6b (varones) del Anexo.

Puede constatarse que no hay efectos significativos del tipo de unión sobre el cuidado de personas, pero sí aparecen brechas en los otros tipos de TDNR. Los casados y las casadas destinan más tiempo que los unidos y las unidas, respectivamente, a las tareas de apoyo escolar. Pero lo que destaca de los cuadros comentados es la diferencia en los quehaceres domésticos, pues en este rubro se refleja claramente la brecha según el tipo de unión: las mujeres casadas destinan más de una hora a tareas de este tipo que las unidas, mientras que no se encuentran diferencias significativas entre los hombres. Puede afirmarse, entonces, que la evidencia va a favor de la especialización y a una sobrecarga laboral sobre las mujeres, principalmente de las 
casadas. Éstas destinan más de una hora a quehaceres domésticos respecto a sus pares unidas.

El resto de las variables permite precisar algunas de las hipótesis que quedaban sugeridas por los resultados anteriores. En primer lugar, son las mujeres las que se ocupan de las tareas específicas de cuidado cuando hay niñas, niños y personas mayores en el hogar. Segundo, el fuerte efecto de la presencia de niñas y niños sobre los quehaceres domésticos apoya la hipótesis del concepto amplio de cuidado: no implica solamente cuidado en sí, sino también la realización de otros tipos de TDNR, por ejemplo, quehaceres generales (lavar, cocinar, etc.) y apoyo escolar.

\section{Consideraciones finales}

A lo largo de este trabajo se presentó y discutió evidencia que da cuenta de la división del trabajo dentro de los hogares en los que residen las personas con pareja en Argentina. El trabajo remunerado es más frecuente entre los hombres y son éstos los que trabajan mayor número de horas por semana; sin embargo, al considerar las tareas domésticas no remuneradas (quehaceres domésticos, apoyo escolar y cuidado), se observa que son más frecuentes entre las mujeres y que son ellas quienes más las absorben, en comparación con los hombres, lo cual es acorde con la evidencia internacional en la materia. Si se consideran ambas actividades, es clara la sobrecarga de trabajo entre las mujeres, a pesar de que la fuente de datos utilizada no permitió captar la realización de actividades simultáneas.

Se puede afirmar que existe la división sexual del trabajo en el interior de los hogares en Argentina y que se observa con independencia del tipo de unión: tanto los hombres casados legalmente como los unidos participan más en las tareas remuneradas y lo hacen mayor número de horas que las mujeres casadas, como así también participan menos y con un número menor de horas en las TDNR. Los resultados encontrados y discutidos en este artículo concuerdan con lo encontrado por Sánchez Peña y Pérez Amador (2016) para México. No se verificaron aquí diferencias de participación en TDR entre los hombres casados y los unidos, y sólamente hay una disparidad te- 
nue en las tareas de apoyo escolar y cuidado. En todo caso, no parece haber especialización entre los hombres, pero sí entre las mujeres.

Estos hallazgos son muy importantes para pensar políticas que tienden a maximizar los beneficios del dividendo de género (DG), una gran esperanza de todos los países de la región. Si esta división de trabajo, y consecuente especialización de tareas, se mantiene o profundiza, la tendencia de las variables condicionantes de la tasa de actividad, como la educación de las mujeres y la fecundidad, no provocará el efecto que se espera para una expansión de la tasa de actividad todavía demasiado reducida, a juzgar por los estándares internacionales. Del análisis precedente, queda muy claro que la dedicación de las mujeres a las TDNR impacta más en sus ofertas de trabajo (incidencia e intensidad) que en la de sus pares masculinos. El efecto es el doble (o más), lo que descubre la división del trabajo subyacente y la especialización de las mujeres en las tareas domésticas, y de los hombres en las remuneradas.

Sería muy útil contar con datos más detallados de la convivencia (y de la extensión del tiempo de convivencia) de las parejas antes del casamiento, por ejemplo. Baxter (2005) muestra que las parejas que han convivido antes del matrimonio tienen una división del trabajo más igualitaria que las que no han cohabitado antes del matrimonio. Esto es importante en la medida en que aquí se obtienen conclusiones del promedio de participación y horas de unidas/os y se compara con el promedio de casadas/os. De darse lo que plantea Baxter, el promedio de casados podría estar afectado por la convivencia previa al matrimonio, algo que no se puede controlar con los datos aquí empleados. 


\section{Anexo}

\section{Cuadro A1}

Definición de las variables y estadísticos descriptivos de la muestra utilizada en las regresiones

\begin{tabular}{|c|c|c|c|c|}
\hline Definición & $\begin{array}{c}\text { Unidad } \\
\text { de medida }\end{array}$ & Todos & Hombres & Mujeres \\
\hline $\begin{array}{l}\text { Participa en mercado } \\
\text { laboral }\end{array}$ & Dummy & 0.750 & 0.944 & 0.563 \\
\hline Participa en TDNR & Dummy & 0.781 & 0.608 & 0.946 \\
\hline Participa en AD & Dummy & 0.727 & 0.506 & 0.936 \\
\hline Participa en $\mathrm{AE}=1$ & Dummy & 0.281 & 0.146 & 0.412 \\
\hline Participa en $\mathrm{TC}=1$ & Dummy & 0.339 & 0.248 & 0.426 \\
\hline Horas en mercado laboral & $\begin{array}{l}\text { Horas / } \\
\text { semana }\end{array}$ & 40.8 & 46.0 & 31.9 \\
\hline Horas TDNR* & Horas / día & 4.833 & 2.285 & 7.248 \\
\hline Horas AD* & Horas / día & 2.552 & 1.133 & 3.892 \\
\hline Horas AE* & Horas / día & 0.566 & 0.263 & 0.860 \\
\hline Horas $\mathrm{TC}^{*}$ & Horas /día & 1.715 & 0.889 & 2.496 \\
\hline Casado $=1$ & Dummy & 0.571 & 0.563 & 0.580 \\
\hline \multicolumn{5}{|l|}{ Unido $=0$} \\
\hline Edad 18-29 & Dummy & 0.178 & 0.150 & 0.205 \\
\hline Edad 30-39 & Dummy & 0.282 & 0.282 & 0.282 \\
\hline Edad 40-49 & Referencia & & & \\
\hline Edad 50-59 & Dummy & 0.211 & 0.226 & 0.197 \\
\hline Edad 60-64 & Dummy & 0.085 & 0.093 & 0.078 \\
\hline Educación: menos de 7 años & Referencia & & & \\
\hline Educación: 7-11 años & Dummy & 0.412 & 0.444 & 0.382 \\
\hline Educación: 12-16 años & Dummy & 0.343 & 0.337 & 0.349 \\
\hline Educación: 17 años o más & Dummy & 0.168 & 0.139 & 0.196 \\
\hline Niños/as, 0-4 años & Cantidad & 0.431 & 0.440 & 0.423 \\
\hline Niños/as, 5-12 años & Cantidad & 0.641 & 0.650 & 0.633 \\
\hline Niños/as, 13-17 años & Cantidad & 0.409 & 0.416 & 0.402 \\
\hline Mayores, 75-79 & Cantidad & 0.015 & 0.013 & 0.016 \\
\hline Mayores, 80 y más & Cantidad & 0.021 & 0.019 & 0.022 \\
\hline
\end{tabular}




\section{Cuadro A1}

(concluye)

\begin{tabular}{llccc}
\hline Definición & $\begin{array}{c}\text { Unidad } \\
\text { de medida }\end{array}$ & Todos & Hombres & Mujeres \\
\hline Gran Buenos Aires=0 & & & & \\
Noroeste Argentino=1 & Dummy & 0.219 & 0.218 & 0.220 \\
Nordeste Argentino=1 & Dummy & 0.132 & 0.130 & 0.133 \\
Cuyo=1 & Dummy & 0.112 & 0.112 & 0.112 \\
Centro=1 & Dummy & 0.210 & 0.211 & 0.210 \\
Sur =1 & Dummy & 0.163 & 0.164 & 0.162 \\
Ingreso no propio & \$ por mes & 6013.5 & 4394.4 & 7539.6 \\
Tamaño muestra & & 31806 & 15438 & 16368 \\
Muestra expandida & & 13448127 & 6588832 & 6859295 \\
\hline
\end{tabular}

* Incluye ceros.

Notas: $\mathrm{TDNR}=$ trabajo doméstico no remunerado; $\mathrm{AD}=$ actividades o quehaceres domésticos; $\mathrm{AE}=$ apoyo escolar; $\mathrm{TC}=$ tareas de cuidado.

Fuente: Construcción propia con base en INDEC, MUT-EAUH.

\section{Cuadro A2}

Funciones probit de participación en el mercado laboral

\begin{tabular}{|c|c|c|c|}
\hline & Ambos sexos & Hombres & Mujeres \\
\hline Variables & (1) & (2) & (3) \\
\hline \multirow[t]{2}{*}{ Persona casada } & $-0.154 * * *$ & -0.027 & $-0.148 * * *$ \\
\hline & $(0.018)$ & $(0.042)$ & $(0.024)$ \\
\hline \multirow[t]{2}{*}{ Edad (años) } & $0.150 * * *$ & $0.151 * * *$ & $0.157 * * *$ \\
\hline & $(0.005)$ & $(0.012)$ & $(0.007)$ \\
\hline \multirow[t]{2}{*}{ Edad al cuadrado } & $-0.002 * * *$ & $-0.002 * * *$ & $-0.002 * * *$ \\
\hline & $(0.000)$ & $(0.000)$ & $(0.000)$ \\
\hline \multirow[t]{2}{*}{ Años escolaridad } & $0.066^{* * *}$ & $0.038 * * *$ & $0.103^{* * *}$ \\
\hline & $(0.002)$ & $(0.005)$ & $(0.003)$ \\
\hline \multirow[t]{2}{*}{ Niñas/os, 0-4 } & $-0.105^{* * *}$ & $0.207 * * *$ & $-0.234 * * *$ \\
\hline & $(0.016)$ & $(0.050)$ & $(0.022)$ \\
\hline \multirow[t]{2}{*}{ Niñas/os, 5-12 } & $-0.071 * * *$ & 0.041 & $-0.106 * * *$ \\
\hline & $(0.011)$ & $(0.027)$ & $(0.014)$ \\
\hline
\end{tabular}




\section{Cuadro A2}

(concluye)

\begin{tabular}{|c|c|c|c|}
\hline & Ambos sexos & Hombres & Mujeres \\
\hline Variables & (1) & (2) & (3) \\
\hline \multirow[t]{2}{*}{ Niñas/os, 13-17 } & $-0.023^{*}$ & 0.005 & $-0.032 *$ \\
\hline & $(0.012)$ & $(0.028)$ & $(0.017)$ \\
\hline \multirow[t]{2}{*}{ Mayores, 70-74 } & $-0.230 * * *$ & -0.151 & $-0.125^{*}$ \\
\hline & $(0.050)$ & $(0.121)$ & $(0.064)$ \\
\hline \multirow[t]{2}{*}{ Mayores, 75-79 } & -0.095 & -0.145 & -0.108 \\
\hline & $(0.063)$ & $(0.139)$ & $(0.082)$ \\
\hline \multirow[t]{2}{*}{ Mayores, 80 y más } & -0.053 & 0.041 & $-0.159 * *$ \\
\hline & $(0.052)$ & $(0.109)$ & $(0.070)$ \\
\hline \multirow[t]{2}{*}{ Ingreso no propio } & $-0.000 * * *$ & $-0.000 * *$ & $-0.000 * * *$ \\
\hline & $(0.000)$ & $(0.000)$ & $(0.000)$ \\
\hline \multirow[t]{2}{*}{ Región NOA } & $-0.090 * * *$ & $-0.168 * * *$ & $-0.077 * *$ \\
\hline & $(0.026)$ & $(0.060)$ & $(0.035)$ \\
\hline \multirow[t]{2}{*}{ Región NEA } & $-0.275 * * *$ & $-0.431 * * *$ & $-0.266 * * *$ \\
\hline & $(0.029)$ & $(0.064)$ & $(0.040)$ \\
\hline \multirow[t]{2}{*}{ Región Cuyo } & $-0.122 * * *$ & -0.007 & $-0.169 * * *$ \\
\hline & $(0.031)$ & $(0.073)$ & $(0.041)$ \\
\hline \multirow[t]{2}{*}{ Región Centro } & 0.013 & -0.056 & 0.035 \\
\hline & $(0.027)$ & $(0.062)$ & $(0.035)$ \\
\hline \multirow[t]{2}{*}{ Región Sur } & 0.031 & $-0.258 * * *$ & 0.033 \\
\hline & $(0.029)$ & $(0.063)$ & $(0.038)$ \\
\hline \multirow[t]{2}{*}{ Ordenada al origen } & $-2.492 * * *$ & $-0.900 * * *$ & $-3.613 * * *$ \\
\hline & $(0.104)$ & $(0.243)$ & $(0.142)$ \\
\hline Pseudo- $\mathrm{R}^{2}$ & 0.086 & 0.183 & 0.122 \\
\hline $\mathrm{LR} \chi^{2}$ & $3096.9 * * *$ & $1363.8^{* * *}$ & $2654.1 * * *$ \\
\hline Observaciones & 30416 & 14744 & 15672 \\
\hline
\end{tabular}

Nota: Se muestran entre paréntesis los errores estándar. Los asteriscos indican significativamente diferente de cero al: *10\%,**5\%,***1\%. La ausencia de asterisco indica que no se puede rechazar la hipótesis de ausencia de relación.

Fuente: Cálculos propios con datos de INDEC, EAHU-MUT. 


\section{Cuadro A3}

Determinantes de las horas de trabajo

\begin{tabular}{|c|c|c|c|}
\hline & Ambos sexos & Hombres & Mujeres \\
\hline Variables & (1) & (2) & (3) \\
\hline \multirow[t]{2}{*}{ Persona casada } & -0.027 & $0.723 * *$ & $-0.895 * *$ \\
\hline & $(0.266)$ & $(0.306)$ & $(0.431)$ \\
\hline \multirow[t]{2}{*}{ Edad (años) } & $0.319 * * *$ & $0.527 * * *$ & 0.114 \\
\hline & $(0.087)$ & $(0.097)$ & $(0.149)$ \\
\hline \multirow[t]{2}{*}{ Edad al cuadrado } & $-0.004 * * *$ & $-0.007 * * *$ & -0.001 \\
\hline & $(0.001)$ & $(0.001)$ & $(0.002)$ \\
\hline \multirow[t]{2}{*}{ Años escolaridad } & $-0.334 * * *$ & $-0.157 * * *$ & -0.071 \\
\hline & $(0.030)$ & $(0.035)$ & $(0.048)$ \\
\hline \multirow[t]{2}{*}{ Niñas/os, 0-4 } & $0.587 * *$ & 0.099 & $-0.849 * *$ \\
\hline & $(0.235)$ & $(0.260)$ & $(0.416)$ \\
\hline \multirow[t]{2}{*}{ Niñas/os, 5-12 } & 0.144 & $0.410 * *$ & $-0.811 * * *$ \\
\hline & $(0.158)$ & $(0.177)$ & $(0.269)$ \\
\hline \multirow[t]{2}{*}{ Niñas/os, 13-17 } & -0.021 & 0.289 & -0.331 \\
\hline & $(0.186)$ & $(0.210)$ & $(0.312)$ \\
\hline \multirow[t]{2}{*}{ Mayores, 70-74 } & -0.026 & -0.132 & 0.350 \\
\hline & $(0.859)$ & (1.049) & $(1.266)$ \\
\hline \multirow[t]{2}{*}{ Mayores, 75-79 } & 0.337 & -0.496 & 0.214 \\
\hline & $(0.996)$ & (1.181) & $(1.528)$ \\
\hline \multirow[t]{2}{*}{ Mayores, 80 y más } & 1.125 & 0.756 & 0.535 \\
\hline & $(0.866)$ & $(1.006)$ & $(1.372)$ \\
\hline \multirow[t]{2}{*}{ Ingreso no propio } & $-0.000 * * *$ & -0.000 & 0.000 \\
\hline & $(0.000)$ & $(0.000)$ & $(0.000)$ \\
\hline \multirow[t]{2}{*}{ Región NOA } & -0.195 & -0.565 & 0.936 \\
\hline & $(0.387)$ & $(0.447)$ & $(0.622)$ \\
\hline \multirow[t]{2}{*}{ Región NEA } & 0.693 & -0.010 & $2.418 * * *$ \\
\hline & $(0.447)$ & $(0.511)$ & $(0.731)$ \\
\hline \multirow[t]{2}{*}{ Región Cuyo } & $1.553^{* * *}$ & $1.610 * * *$ & 0.974 \\
\hline & $(0.459)$ & $(0.525)$ & $(0.749)$ \\
\hline
\end{tabular}




\section{Cuadro A3}

(concluye)

\begin{tabular}{|c|c|c|c|}
\hline & Ambos sexos & Hombres & Mujeres \\
\hline Variables & (1) & (2) & (3) \\
\hline \multirow{2}{*}{ Región Centro } & 0.006 & -0.262 & 0.638 \\
\hline & $(0.386)$ & $(0.448)$ & $(0.613)$ \\
\hline \multirow[t]{2}{*}{ Región Sur } & $2.102 * * *$ & $2.133^{* * *}$ & $1.325^{* *}$ \\
\hline & $(0.415)$ & $(0.482)$ & $(0.661)$ \\
\hline \multirow[t]{2}{*}{ Ordenada al origen } & $39.291 * * *$ & $37.930 * * *$ & $31.637 * * *$ \\
\hline & $(1.738)$ & $(1.964)$ & $(2.936)$ \\
\hline $\mathrm{R}^{2-}$ ajustado & 0.019 & 0.014 & 0.003 \\
\hline Estadístico F & $25.9 * * *$ & $12.6^{* * *}$ & $2.3 * * *$ \\
\hline Observaciones & 20354 & 12949 & 7405 \\
\hline
\end{tabular}

Nota: Se muestran entre paréntesis los errores estándar. Los asteriscos indican significativamente diferente de cero al: $* 10 \%, * * 5 \%, * * * 1 \%$. La ausencia de asterisco indica que no se puede rechazar la hipótesis de ausencia de relación.

Fuente: Cálculos propios con datos de INDEC, EAHU-MUT.

\section{Cuadro A4}

Funciones probit de participación

en tareas domésticas no remuneradas

\begin{tabular}{lcccc}
\hline \multirow{2}{*}{ Variables } & Ambos sexos & & Hombres & Mujeres \\
\cline { 2 - 3 } Persona casada & $(\mathbf{1})$ & $(2)$ & $(3)$ \\
Edad (años) & 0.028 & -0.022 & -0.015 \\
& $(0.019)$ & $(0.024)$ & $(0.042)$ \\
Edad al cuadrado & -0.002 & $0.014^{*}$ & 0.013 \\
& $(0.006)$ & $(0.007)$ & $(0.012)$ \\
Años escolaridad & -0.000 & $-0.000^{* *}$ & -0.000 \\
& $(0.000)$ & $(0.000)$ & $(0.000)$ \\
Niñas/os, 0-4 & $0.015^{* * * *}$ & $0.023^{* * *}$ & $-0.015^{* * *}$ \\
Niñas/os, 5-12 & $(0.002)$ & $(0.003)$ & $(0.004)$ \\
& $0.172^{* * * *}$ & $0.198^{* * *}$ & $0.390^{* * *}$ \\
& $(0.017)$ & $(0.021)$ & $(0.050)$ \\
& $0.073^{* * *}$ & $0.066^{* * *}$ & $0.188^{* * *}$ \\
& $(0.011)$ & $(0.014)$ & $(0.030)$
\end{tabular}




\section{Cuadro A4}

(concluye)

\begin{tabular}{|c|c|c|c|}
\hline & Ambos sexos & Hombres & Mujeres \\
\hline Variables & (1) & (2) & (3) \\
\hline \multirow[t]{2}{*}{ Niñas/os, 13-17 } & $-0.048 * * *$ & $-0.086^{* * *}$ & 0.035 \\
\hline & $(0.013)$ & $(0.016)$ & $(0.030)$ \\
\hline \multirow[t]{2}{*}{ Mayores, 70-74 } & $0.178 * * *$ & 0.028 & 0.059 \\
\hline & $(0.056)$ & $(0.080)$ & $(0.102)$ \\
\hline \multirow[t]{2}{*}{ Mayores, 75-79 } & 0.004 & 0.026 & -0.021 \\
\hline & $(0.067)$ & $(0.091)$ & $(0.125)$ \\
\hline \multirow[t]{2}{*}{ Mayores, 80 y más } & -0.040 & -0.035 & 0.075 \\
\hline & $(0.055)$ & $(0.073)$ & $(0.113)$ \\
\hline \multirow[t]{2}{*}{ Ingreso no propio } & $0.000 * * *$ & $0.000 *$ & $-0.000 *$ \\
\hline & $(0.000)$ & $(0.000)$ & $(0.000)$ \\
\hline \multirow[t]{2}{*}{ Región NOA } & -0.033 & $-0.095 * * *$ & 0.047 \\
\hline & $(0.027)$ & $(0.035)$ & $(0.056)$ \\
\hline \multirow[t]{2}{*}{ Región NEA } & $0.130 * * *$ & $0.096 * *$ & 0.090 \\
\hline & $(0.031)$ & $(0.040)$ & $(0.066)$ \\
\hline \multirow[t]{2}{*}{ Región Cuyo } & $0.123 * * *$ & 0.065 & $0.416 * * *$ \\
\hline & $(0.032)$ & $(0.041)$ & $(0.080)$ \\
\hline \multirow[t]{2}{*}{ Región Centro } & -0.032 & $-0.066^{*}$ & 0.041 \\
\hline & $(0.027)$ & $(0.035)$ & $(0.056)$ \\
\hline \multirow[t]{2}{*}{ Región Sur } & -0.025 & 0.031 & -0.002 \\
\hline & $(0.029)$ & $(0.037)$ & $(0.060)$ \\
\hline \multirow[t]{2}{*}{ Ordenada al origen } & $0.599 * * *$ & -0.190 & $1.399 * * *$ \\
\hline & $(0.112)$ & $(0.149)$ & $(0.230)$ \\
\hline Pseudo- $\mathrm{R}^{2}$ & 0.027 & -0.020 & -0.040 \\
\hline LR $\chi^{2}$ & $30.4 * * *$ & $14.8 * * *$ & $234.6 * * *$ \\
\hline Observaciones & 30423 & 14755 & 15668 \\
\hline
\end{tabular}

Nota: Se muestran entre paréntesis los errores estándar. Los asteriscos indican significativamente diferente de cero al: $* 10 \%, * * 5 \%, * * * 1 \%$. La ausencia de asterisco indica que no se puede rechazar la hipótesis de ausencia de relación.

Fuente: Cálculos propios con datos de INDEC, EAHU-MUT. 


\section{Cuadro A5}

Determinantes de las horas de TDNR

\begin{tabular}{|c|c|c|c|}
\hline & Ambos sexos & Hombres & Mujeres \\
\hline Variables & (1) & (2) & (3) \\
\hline \multirow[t]{2}{*}{ Persona casada } & $2.454 * * *$ & 0.154 & $1.914 * * *$ \\
\hline & $(0.413)$ & $(0.385)$ & $(0.582)$ \\
\hline \multirow[t]{2}{*}{ Edad (años) } & $-0.886^{* * *}$ & 0.097 & -0.233 \\
\hline & $(0.121)$ & $(0.118)$ & $(0.168)$ \\
\hline \multirow[t]{2}{*}{ Edad al cuadrado } & $0.005^{* * *}$ & -0.002 & -0.002 \\
\hline & $(0.001)$ & $(0.001)$ & $(0.002)$ \\
\hline \multirow[t]{2}{*}{ Años escolaridad } & $-0.117 * *$ & $0.289 * * *$ & $-0.699 * * *$ \\
\hline & $(0.046)$ & $(0.044)$ & $(0.064)$ \\
\hline \multirow[t]{2}{*}{ Niñas/os, 0-4 } & $11.077 * * *$ & $6.029 * * *$ & $17.063 * * *$ \\
\hline & $(0.360)$ & $(0.334)$ & $(0.511)$ \\
\hline \multirow[t]{2}{*}{ Niñas/os, 5-12 } & $5.847 * * *$ & $2.519 * * *$ & $8.328 * * *$ \\
\hline & $(0.241)$ & $(0.225)$ & $(0.341)$ \\
\hline \multirow[t]{2}{*}{ Niñas/os, 13-17 } & $0.716^{* *}$ & $-0.916 * * *$ & $1.307 * * *$ \\
\hline & $(0.283)$ & $(0.264)$ & $(0.401)$ \\
\hline \multirow[t]{2}{*}{ Mayores, 70-74 } & $5.269 * * *$ & 0.760 & $4.767 * * *$ \\
\hline & $(1.186)$ & (1.288) & $(1.498)$ \\
\hline \multirow[t]{2}{*}{ Mayores, 75-79 } & $4.141 * * *$ & 1.397 & $6.803 * * *$ \\
\hline & $(1.479)$ & $(1.472)$ & (1.969) \\
\hline \multirow[t]{2}{*}{ Mayores, 80 y más } & $5.009 * * *$ & $2.391 * *$ & $8.926 * * *$ \\
\hline & $(1.234)$ & $(1.190)$ & $(1.683)$ \\
\hline \multirow[t]{2}{*}{ Ingreso no propio } & $0.001 * * *$ & $0.000 * * *$ & $0.000 * * *$ \\
\hline & $(0.000)$ & $(0.000)$ & $(0.000)$ \\
\hline \multirow[t]{2}{*}{ Región NOA } & $-2.817 * * *$ & $-3.390 * * *$ & $-3.226 * * *$ \\
\hline & $(0.595)$ & $(0.559)$ & $(0.831)$ \\
\hline \multirow[t]{2}{*}{ Región NEA } & $-3.922 * * *$ & $-2.063 * * *$ & $-8.295 * * *$ \\
\hline & $(0.674)$ & $(0.635)$ & $(0.940)$ \\
\hline \multirow[t]{2}{*}{ Región Cuyo } & -0.752 & $-2.750 * * *$ & 0.122 \\
\hline & $(0.701)$ & $(0.660)$ & $(0.978)$ \\
\hline \multirow[t]{2}{*}{ Región Centro } & $-1.878 * * *$ & $-2.034 * * *$ & $-1.903 * *$ \\
\hline & $(0.598)$ & $(0.563)$ & $(0.835)$ \\
\hline
\end{tabular}




\begin{tabular}{|c|c|c|c|}
\hline & Ambos sexos & Hombres & Mujeres \\
\hline Variables & (1) & (2) & (3) \\
\hline \multirow[t]{2}{*}{ Región Sur } & $-5.136^{* * *}$ & -0.911 & $-6.663 * * *$ \\
\hline & $(0.640)$ & $(0.601)$ & $(0.896)$ \\
\hline \multirow[t]{2}{*}{ Ordenada al origen } & $49.363 * * *$ & $10.707 * * *$ & $60.188 * * *$ \\
\hline & $(2.442)$ & $(2.405)$ & $(3.314)$ \\
\hline $\mathrm{R}^{2-}$ ajustado & 0.121 & 0.058 & 0.192 \\
\hline Estadístico F & $259.3 * * *$ & $56.4 * * *$ & $232.8 * * *$ \\
\hline Observaciones & 30291 & 14689 & 15602 \\
\hline
\end{tabular}

Nota: Se muestran entre paréntesis los errores estándar. Los asteriscos indican significativamente diferente de cero al: $* 10 \%, * * 5 \%, * * * 1 \%$. La ausencia de asterisco indica que no se puede rechazar la hipótesis de ausencia de relación.

Fuente: Cálculos propios con datos de INDEC,EAHU-MUT.

\section{Cuadro A6a}

Determinantes de las horas de TDNR, distintas actividades. Mujeres

\begin{tabular}{|c|c|c|c|}
\hline & $\begin{array}{l}\text { Quehaceres } \\
\text { domésticos }\end{array}$ & Apoyo escolar & $\begin{array}{c}\text { Cuidado de } \\
\text { personas }\end{array}$ \\
\hline Variables & (1) & (2) & (3) \\
\hline \multirow[t]{2}{*}{ Persona casada } & $1.198 * * *$ & $0.234^{*}$ & 0.454 \\
\hline & $(0.294)$ & $(0.131)$ & $(0.431)$ \\
\hline \multirow[t]{2}{*}{ Edad (años) } & $0.496 * * *$ & $0.212 * * *$ & $-0.939 * * *$ \\
\hline & $(0.085)$ & $(0.038)$ & $(0.124)$ \\
\hline \multirow[t]{2}{*}{ Edad al cuadrado } & $-0.005 * * *$ & $-0.003 * * *$ & $0.006^{* * *}$ \\
\hline & $(0.001)$ & $(0.000)$ & $(0.001)$ \\
\hline \multirow[t]{2}{*}{ Años escolaridad } & $-0.489 * * *$ & -0.006 & $-0.205 * * *$ \\
\hline & $(0.032)$ & $(0.015)$ & $(0.048)$ \\
\hline \multirow[t]{2}{*}{ Niñas/os, 0-4 } & $3.334 * * *$ & $0.460 * * *$ & $13.253 * * *$ \\
\hline & $(0.258)$ & $(0.115)$ & $(0.379)$ \\
\hline \multirow[t]{2}{*}{ Niñas/os, 5-12 } & $1.463 * * *$ & $2.631 * * *$ & $4.227 * * *$ \\
\hline & $(0.173)$ & $(0.077)$ & $(0.253)$ \\
\hline \multirow[t]{2}{*}{ Niñas/os, 13-17 } & $0.564 * * *$ & $0.619 * * *$ & 0.142 \\
\hline & $(0.203)$ & $(0.091)$ & $(0.297)$ \\
\hline
\end{tabular}




\section{Cuadro A6a}

(concluye)

\begin{tabular}{|c|c|c|c|}
\hline & $\begin{array}{c}\text { Quehaceres } \\
\text { domésticos }\end{array}$ & Apoyo escolar & $\begin{array}{c}\text { Cuidado de } \\
\text { personas }\end{array}$ \\
\hline Variables & (1) & (2) & (3) \\
\hline \multirow[t]{2}{*}{ Mayores, 70-74 } & 0.032 & $1.390 * * *$ & $3.332 * * *$ \\
\hline & $(0.757)$ & $(0.338)$ & $(1.110)$ \\
\hline \multirow[t]{2}{*}{ Mayores, 75-79 } & -0.326 & 0.234 & $6.840 * * *$ \\
\hline & $(0.993)$ & $(0.444)$ & $(1.460)$ \\
\hline \multirow[t]{2}{*}{ Mayores de 80 y más } & 0.074 & 0.424 & $8.384 * * *$ \\
\hline & $(0.851)$ & $(0.380)$ & $(1.247)$ \\
\hline \multirow[t]{2}{*}{ Ingreso no propio } & $0.000 * * *$ & 0.000 & $0.000 * * *$ \\
\hline & $(0.000)$ & $(0.000)$ & $(0.000)$ \\
\hline \multirow[t]{2}{*}{ Región NOA } & $-3.298 * * *$ & $0.526^{* * *}$ & -0.439 \\
\hline & $(0.420)$ & $(0.188)$ & $(0.616)$ \\
\hline \multirow[t]{2}{*}{ Región NEA } & $-3.708 * * *$ & $-0.787 * * *$ & $-3.786 * * *$ \\
\hline & $(0.475)$ & $(0.212)$ & $(0.697)$ \\
\hline \multirow[t]{2}{*}{ Región Cuyo } & -0.668 & 0.213 & 0.594 \\
\hline & $(0.494)$ & $(0.221)$ & $(0.725)$ \\
\hline \multirow[t]{2}{*}{ Región Centro } & $-2.352 * * *$ & 0.251 & 0.221 \\
\hline & $(0.422)$ & $(0.189)$ & $(0.619)$ \\
\hline \multirow[t]{2}{*}{ Región Sur } & $-4.235 * * *$ & $-0.526^{* * *}$ & $-1.915 * * *$ \\
\hline & $(0.453)$ & $(0.202)$ & $(0.664)$ \\
\hline \multirow[t]{2}{*}{ Ordenada al origen } & $20.308 * * *$ & -0.899 & $40.756 * * *$ \\
\hline & $(1.675)$ & $(0.749)$ & $(2.457)$ \\
\hline $\mathrm{R}^{2-}$ ajustado & 0.045 & 0.133 & 0.207 \\
\hline Estadístico F & $46.8^{* * *}$ & $15.6^{* * *}$ & $255.9 * * *$ \\
\hline Observaciones & 15609 & 15613 & 15612 \\
\hline
\end{tabular}

Nota: Se muestran entre paréntesis los errores estándar. Los asteriscos indican significativamente diferente de cero al: $* 10 \%, * * 5 \%, * * * 1 \%$. La ausencia de asterisco indica que no se puede rechazar la hipótesis de ausencia de relación.

Fuente: Cálculos propios con datos de INDEC, EAHU-MUT. 


\section{Cuadro A6b}

Determinantes de las horas de TDNR.

Distintas actividades. Varones

\begin{tabular}{|c|c|c|c|}
\hline & $\begin{array}{l}\text { Quehaceres } \\
\text { domésticos }\end{array}$ & Apoyo escolar & $\begin{array}{c}\text { Cuidado de } \\
\text { personas }\end{array}$ \\
\hline Variables & (1) & (2) & (3) \\
\hline \multirow[t]{2}{*}{ Persona casada } & -0.202 & $0.217 * *$ & 0.133 \\
\hline & $(0.202)$ & $(0.085)$ & $(0.263)$ \\
\hline \multirow[t]{2}{*}{ Edad (años) } & $0.136^{* *}$ & $0.055^{* *}$ & -0.093 \\
\hline & $(0.062)$ & $(0.026)$ & $(0.081)$ \\
\hline \multirow[t]{2}{*}{ Edad al cuadrado } & -0.001 & $-0.001 * * *$ & -0.000 \\
\hline & $(0.001)$ & $(0.000)$ & $(0.001)$ \\
\hline \multirow[t]{2}{*}{ Años escolaridad } & $0.085 * * *$ & $0.046^{* * *}$ & $0.158 * * *$ \\
\hline & $(0.023)$ & $(0.010)$ & $(0.030)$ \\
\hline \multirow[t]{2}{*}{ Niñas/os, 0-4 } & $0.650 * * *$ & $0.314^{* * *}$ & $5.060 * * *$ \\
\hline & $(0.175)$ & $(0.074)$ & $(0.228)$ \\
\hline \multirow[t]{2}{*}{ Niñas/os, 5-12 } & 0.051 & $0.797 * * *$ & $1.670 * * *$ \\
\hline & $(0.118)$ & $(0.050)$ & $(0.153)$ \\
\hline \multirow[t]{2}{*}{ Niñas/os, 13-17 } & $-0.411 * * *$ & $0.140 * *$ & $-0.641 * * *$ \\
\hline & $(0.138)$ & $(0.058)$ & $(0.180)$ \\
\hline \multirow[t]{2}{*}{ Mayores, 70-74 } & $-1.220 *$ & $0.586^{* *}$ & 1.396 \\
\hline & $(0.674)$ & $(0.285)$ & $(0.880)$ \\
\hline \multirow[t]{2}{*}{ Mayores, 75-79 } & 0.608 & -0.319 & 1.111 \\
\hline & $(0.771)$ & $(0.326)$ & $(1.006)$ \\
\hline \multirow[t]{2}{*}{ Mayores, 80 y más } & -0.581 & 0.082 & $2.893 * * *$ \\
\hline & $(0.623)$ & $(0.263)$ & $(0.813)$ \\
\hline \multirow[t]{2}{*}{ Ingreso no propio } & $0.000 * * *$ & 0.000 & $0.000 * * *$ \\
\hline & $(0.000)$ & $(0.000)$ & $(0.000)$ \\
\hline \multirow[t]{2}{*}{ Región NOA } & $-1.706^{* * *}$ & $-0.341 * * *$ & $-1.339 * * *$ \\
\hline & $(0.293)$ & $(0.124)$ & $(0.382)$ \\
\hline \multirow[t]{2}{*}{ Región NEA } & -0.133 & $-0.265^{*}$ & $-1.662 * * *$ \\
\hline & $(0.333)$ & $(0.141)$ & $(0.434)$ \\
\hline \multirow[t]{2}{*}{ Región Cuyo } & $-1.252 * * *$ & $-0.300 * *$ & $-1.194 * * *$ \\
\hline & $(0.346)$ & $(0.146)$ & $(0.451)$ \\
\hline
\end{tabular}




\section{Cuadro A6b}

(concluye)

\begin{tabular}{|c|c|c|c|}
\hline & $\begin{array}{c}\text { Quehaceres } \\
\text { domésticos }\end{array}$ & Apoyo escolar & $\begin{array}{c}\text { Cuidado de } \\
\text { personas }\end{array}$ \\
\hline Variables & (1) & (2) & (3) \\
\hline \multirow[t]{2}{*}{ Región Centro } & $-1.200 * * *$ & $-0.287 * *$ & -0.553 \\
\hline & $(0.295)$ & $(0.125)$ & $(0.385)$ \\
\hline \multirow[t]{2}{*}{ Región Sur } & $-0.714 * *$ & 0.073 & -0.281 \\
\hline & $(0.315)$ & $(0.133)$ & $(0.410)$ \\
\hline \multirow[t]{2}{*}{ Ordenada al origen } & $4.187 * * *$ & -0.579 & $7.110 * * *$ \\
\hline & $(1.260)$ & $(0.533)$ & $(1.643)$ \\
\hline $\mathrm{R}^{2-}$ ajustado & 0.007 & 0.032 & 0.080 \\
\hline Estadístico F & $7.8 * * *$ & $31.5 * * *$ & $81.2 * * *$ \\
\hline Observaciones & 14692 & 14698 & 14697 \\
\hline
\end{tabular}

Nota: Se muestran entre paréntesis los errores estándar. Los asteriscos indican significativamente diferente de cero al: $* 10 \%, * * 5 \%, * * * 1 \%$. La ausencia de asterisco indica que no se puede rechazar la hipótesis de ausencia de relación.

Fuente: Cálculos propios con datos de INDEC, EAHU-MUT.

\section{Bibliografía}

Abraham, K. y Mackie, C. (eds.) (2005). Beyond the market: Designing nonmarket accounts for the United States. Panel to study the design of nonmarket accounts. Washington, D.C: National Research Council of the National Academies.

Aguirre, R., García Sainz, C. y Carrasco, C. (2005). El tiempo, los tiempos, una vara de desigualdad. (Documento de trabajo, núm. 65). Serie Mujer y Desarrollo. Santiago de Chile: CEPAL. https: //repositorio.cepal.org/bitstream/handle/11362/5936/1/S0 55367 es.pdf

Arosio, L. (2017). Marriage, cohabitation and participation in domestic labor: Men and women in contemporary Italy. European Scientific Journal, 13(8), 236-249. https://eujournal.org/index. php/esj/article/view/8998/8563

Barg, K. y Beblo, M. (2012). Does "sorting into specialization”. Explain the differences in time use between married and cohabi- 
ting couples? An empirical application for Germany. Annals of Economics and Statistics, 105/106, 127-152. https://ideas.repec. org/a/adr/anecst/y2012i105-106p127-152.html

Baxter, J. (2005). To marry or not to marry: Marital status and the household division of labor. Journal of Family Issues, 26(3), 300-321. https://www.researchgate.net/deref/http $\% 3 \mathrm{~A} \% 2 \mathrm{~F} \% 2 \mathrm{~F}$ dx.doi.org\%2F10.1177\%2F0192513X04270473

Becker, G. (1965). A theory of allocation time. The Economic Journal, 75(299), 493-517. https://www.jstor.org/stable/2228949? seq $=1$ \#metadata_info_tab_contents

Becker, G. (1981). A treatise on the family. Londres: Harvard University Press.

Bianchi, S., Lesnard, L., Nazio, T. y Raley, S. (2014). Gender and time allocation of cohabiting and married women and men in France, Italy, and the United States. Demographic Research, 31(8), 183-216. https://www.demographic-research.org/volumes/vol31 /8/31-8.pdf

Blair, S. y Lichter, T. (1991). Measuring the division of household labor: Gender segregation of housework among American couples. Journal of Family Issues, 12(1), 91-113. https://www.research gate.net/deref/http $\% 3 \mathrm{~A} \% 2 \mathrm{~F} \% 2 \mathrm{Fdx}$.doi.org $\% 2 \mathrm{~F} 10.1177 \%$ 2F019251391012001007

Blau, F., Ferber, M. y Winkler, A. (2014). The economics of the women, men, and work. Londres: Prentice Hall.

Bloemen, H., Pasqua, S. y Stancanelli, E. (2010). An empirical analysis of the time allocation of Italian couples: Are they responsive? Review of Economics of the Household, 8(3), 345-369. https:// link.springer.com/article/10.1007/s11150-009-9083-4

Browning, M., Chiappori, P. y Weiss, Y. (2014). Economics of the family. Nueva York: Cambridge University Press.

Bryant, K. y Zick, C. (2006). The economic organization of the household. Londres: Cambridge University Press.

Cherlin, A. (2004). The deinstitutionalization of American marriage. Journal of Marriage and Family, 66(4), 848-861. https:// onlinelibrary.wiley.com/doi/full/10.1111/j.0022-2445.2004.000 58.x 
Davis, S., Greenstein, T. y Gerteisen, J. (2007). Effects of union type on division of household labor: Do cohabiting men really perform more housework? Journal of Family Issues, 28(9), 12461272. https://www.researchgate.net/deref/http $\% 3 \mathrm{~A} \% 2 \mathrm{~F} \% 2 \mathrm{Fdx}$. doi.org\%2F10.1177\%2F0192513X07300968

Domínguez-Folgueras, M. (2012a). La división del trabajo doméstico en las parejas españolas. Un análisis del uso del tiempo. Revista Internacional de Sociología, 70(1), 153-179. http:// revintsociologia.revistas.csic.es/index.php/revintsociologia/ article/view/439

Domínguez-Folgueras, M. (2012b). Is cohabitation more egalitarian? The division of household labor in five European countries. Journal of Family Issues, 34(12), 1623-1646. https://hal-scien cespo.archives-ouvertes.fr/hal-01024517

Esplen, E. (2009). Género y cuidados: una mirada general. En Breve, Boletín de Bridge, 20. http://americalatinagenera.org/ newsite/images/cdr-documents/publicaciones/enbrevegenero ycuidados2009.pdf

Esquivel, V. (2009). Uso del tiempo en la Ciudad de Buenos Aires. Los Polvorines: Universidad Nacional de General Sarmiento.

García, B. (2019). El trabajo doméstico y de cuidado: su importancia y principales hallazgos en el caso mexicano. Estudios Demográficos y Urbanos, 34(2), 237-267. https://estudiosdemograficosy urbanos.colmex.mx/index.php/edu/article/view/1811/pdf

García, B. y De Oliveira, O. (2007). Trabajo extradoméstico y relaciones de género: una nueva mirada. En M. Gutiérrez (ed.), Género, familias y trabajo: rupturas y continuidades. Desafios para la investigación politica (pp. 49-87). Buenos Aires: Consejo Latinoamericano de Ciencias Sociales.

Gronau, R. (1976). Leisure, home production and work. The theory of allocation of time revisited. Journal of Political Economy, 85(6), 1099-1123.https://www.jstor.org/stable/1837419?seq=1\#metada ta_info_tab_contents

Henkens, K., Grift, Y. y Siegers, J. (2002). Changes in female labour supply in the Netherlands 1989-1998: The case of married and 
cohabiting women. European Journal of Population, 18(1), 3957. https://link.springer.com/article/10.1023/A:1013868021024 Hoem, J. (2005). Why does Sweden have such high fertility? (Documento de Trabajo, WP-2005-009). Alemania: Max Planck Institute for Demographic Research. https://www.demogr.mpg.de/ papers/working/wp-2005-009.pdf

International Labour Office. (ILO). (2017). World employment and social outlook: Trends for women 2017. Ginebra: International Labour Office. https://www.ilo.org/global/research/global-reports /weso/trends-for-women2017/lang--en/index.htm

Killingsworth, R. y Heckman, J. (1986). Female labor supply: A survey. En O. Ashenfelter y R. Layard (eds.), Handbook of labor economics, vol. 1 (pp. 103-204). Amsterdam: North-Holland.

Kuperberg, A. (2012). Reassessing differences in work and income in cohabitation and marriage. Journal of Marriage and Family, 74(4), 688-707. https://www.jstor.org/stable/41678750?seq=1\# metadata_info_tab_contents

Martínez, C., Miller, T. y Saad, P. (2013). Participación laboral femenina y bono de género en América Latina. (Documento de Proyecto, núm. LC/W.570). Santiago de Chile: CEPAL-CELADE. https://repositorio.cepal.org/bitstream/handle/11362/35897/1/ S20131095_es.pdf

Meggiolaro, S. (2014). Household labor allocation among married and cohabiting couples in Italy. Journal of Family Issues, 35(6), 851-876. https://www.researchgate.net/deref/http $\% 3 \mathrm{~A} \% 2 \mathrm{~F} \% 2 \mathrm{~F}$ dx.doi.org\%2F10.1177\%2F0192513X13491409

Paz, J. (2018). Ensayos sobre el bono de género en la Argentina. Factores internos y externos que determinan la participación económica femenina. (Tesis doctoral, Universidad Nacional de Córdoba). https://rdu.unc.edu.ar/handle/11086/6723

Sánchez Peña, L. y Pérez Amador, J. (2016). Distintas o iguales: las diferencias en el trabajo doméstico de las parejas de doble ingreso entre las uniones libres y los matrimonios. Estudios Demográficos y Urbanos, 31(3), 593-634. https://estudiosdemo graficosyurbanos.colmex.mx/index.php/edu/article/view/11/pdf 
Shelton, B. y John, D. (1993). Does marital status make a difference? Journal of Family Issues, 14(3), 401-420. https://journals. sagepub.com/doi/pdf/10.1177/019251393014003004

South, J. y Spitze, G. (1994). Housework in marital and nonmarital households. American Sociological Review, 59(3), 327-347. https: //psycnet.apa.org/record/1995-40239-001

Stewart, J. (2009). Tobit or not tobit? (Documento de Trabajo, núm. 4588). Alemania. Institute of Labor Economics. http://ftp.iza. org/dp4588.pdf

Stiglitz, J., Sen, A. y Fitoussi, J. P. (2009). Report by the Commission on the Measurement of Economic Performance and Social Progress. https://www.researchgate.net/publication/258260767 Report_of_the_Commission_on_the_Measurement_of_ Economic_Performance and Social_Progress_CMEPSP Torns, T. (2004). Las políticas de tiempo: un reto para las políticas del estado de bienestar. Trabajo. Revista Iberoamericana de Relaciones Laborales, 13, 145-164. https://dialnet.unirioja.es/ servlet/articulo?codigo $=1039489$

\section{Acerca del autor}

Jorge A. Paz es doctor en Economía y doctor en Demografía. Se desempeña como investigador principal del Consejo Nacional de Investigaciones Científicas y Técnicas (Conicet) y profesor de la Universidad Nacional de Salta, Argentina. Ha sido investigador asociado en la Comisión Económica para América Latina y el Caribe (CEPAL). Es especialista en temas relacionados con el mercado laboral desde una perspectiva de género y el análisis cuantitativo de la pobreza. ORCID: https://orcid.org/0000-0002-2009-680X

Entre sus publicaciones se encuentran:

Paz, J. (2020). Asignación universal por hijo y privaciones educativas en la Argentina. Población y Sociedad, 27(1), 59-88. http:// dx.doi.org/10.19137/pys-2020-270103

Paz, J. (2019). Envejecimiento demográfico y oferta laboral de hombres y mujeres. Revista de Análisis Económico, 34(2), 53-80. https://www.rae-ear.org/index.php/rae/article/view/635 
Paz, J. y Arévalo, C. (20189). Pobreza en las personas mayores. Un estudio multidimensional para Argentina. Relap, Revista Latinoamericana de Población, 13(25), 75-102. https://doi. org/10.31406/relap2019.v13.i2.n25.4

Recepción: 16 de enero de 2018. Aceptación: 28 de junio de 2019. 
